\title{
RNF4 Is a Coactivator for Nuclear Factor $Y$ on GTP Cyclohydrolase I Proximal Promoter
}

\author{
Sheng-Ming Wu, Wen-Chih Kuo, Wuh-Liang Hwu, Kuo-Yuan Hwa, Roberto Mantovani, and \\ Yu-May Lee
}

Institute of Biological Chemistry (S.-M.W., W.-C.K., K.Y.H., Y.-M.L.), Academia Sinica, Taipei, Taiwan, Republic of China; Institute of Biochemical Science (W.-C.K.), National Taiwan University, Department of Pediatrics and Medical Genetics (W.L.H.), National Taiwan University Hospital and National Taiwan University College of Medicine, Taipei, Taiwan, Republic of China; and Department of Biomolecular Science, University of Milan, Milan, Italy (R.M.)

Received January 28, 2004; accepted August 5, 2004

This article is available online at http://molpharm.aspetjournals.org

\begin{abstract}
GTP cyclohydrolase I (GCH) is the rate-controlling enzyme in the production of tetrahydrobiopterin $(\mathrm{BH} 4)$ that is essential for the synthesis of nitric oxide and catecholamines including dopamine and serotonin. Therefore, the regulation of $\mathrm{GCH}$ expression is important in determining the catecholamine levels in the brain under pathophysiological conditions. During the study of human disease dopa-responsive dystonia, we found that coactivator RNF4 is involved in the $\mathrm{GCH}$ gene expression. Through serial deletion and mutagenesis studies of the $\mathrm{GCH}$ promoter, we defined the RNF4-responsive element on $\mathrm{GCH}$ proximal promoter as a CCAAT box. RNF4 did not possess specific DNA binding activity toward this CCAAT box, which
\end{abstract}

suggests that RNF4 may be a coactivator of the CCAAT boxbinding protein nuclear factor $Y(N F-Y)$. Cotransfection of a dominant-negative mutant of NF-Y resulted in a significant reduction in RNF4-mediated CCAAT box activation. In addition, overexpression of RNF4 could not activate the CCAAT box in Drosophila melanogaster SL2 cells, which are devoid of endogenous NF-Y, whereas overexpression of RNF4 and NF-Y could. Furthermore, immunoprecipitation experiments revealed the physical association between RNF4 and the NF-Y complex. These data indicate that RNF4 imposes functional importance on $\mathrm{GCH}$ promoter.
GTP cyclohydrolase I ( $\mathrm{GCH})$ is the rate-controlling enzyme in the production of tetrahydrobiopterin (BH4) (Blau and Niederwieser, 1985; Nichol et al., 1985) that is essential for the synthesis of nitric oxide and neurotransmitters including dopamine and serotonin. $\mathrm{BH} 4$ was also reported to possess protective effects against nitric oxide or reactive oxygen cytotoxicities in various types of cells (Shimizu et al., 1999). Patterns of GCH expression in tissues indicate both temporal and spatial regulation of this gene (Nichol et al., 1985; Werner et al., 1990; Hirayama and Kapatos, 1998). Therefore, GCH is important in determining the levels of neurotransmitters in the brain under both physiological and pathological conditions.

Hereditary progressive dystonia (HPD) with marked diur-

This work was supported by the National Science Council Grant NSC-90 2311-B-001-199 and NSC-91-2311-B-001-156.

S.-M.W. and W.-C.K. contributed equally to this study. nal fluctuation, also termed Segawa's disease or dopa-responsive dystonia (DRD), is a dominantly inherited postural dystonia with female predominance caused by the mutation of the GCH gene (Ichinose et al., 1994). In HPD/DRD patients with mutations present in only one of the GCH alleles, GCH activity is reduced to $20 \%$ of normal (Ichinose et al., 1994). A dominant-negative (DN) effect, caused by either an abnormal or an unstable GCH subunit, has been used to explain the less than 50\% activity in heterozygous patients (Hirano et al., 1998; Suzuki et al., 1999; Hwu et al., 2000). We have demonstrated this dominant-negative effect for GCH G201E mutant protein in a cell model, in which the GCH mutant reduced the wild-type protein level (Hwu et al., 1999, 2000). We recently identified a cell strain that could not show the $\mathrm{DN}$ effect (the non-DN cells). In these cells, wild-type $\mathrm{GCH}$ protein expression did not change in the presence of $\mathrm{GCH}$ mutant G201E. We have isolated several cDNA clones en-

ABBREVIATIONS: GCH, GTP cyclohydrolase I; BH4, 5,6,7,8- tetrahydrobiopterin; HPD, hereditary progressive dystonia; DRD, dopa-responsive dystonia; GCF, GC-box binding factor; GFP, green fluorescent protein; GST, glutathione S-transferase; ChIP, chromatin immunoprecipitation; CMV, cytomegalovirus; HEK, human embryonic kidney; HA, hemagglutinin; CRE, cAMP response element; bp, base pair(s); kb, kilobase(s); nt, nucleotide; EMSA, electrophoretic mobility shift assay; RT, reverse transcription; PCR, polymerase chain reaction; NF, nuclear factor; CAT, chloramphenicol acetyl transferase; DN, dominant-negative; AGP/EBP, $\alpha-1$ acid glycoprotein/enhancer-binding protein; TBP, TATA-binding protein. 
riched in the non-DN cells by differential display study. RNF4 was one of the clones enriched in the non-DN cells.

RNF4 is a recently identified human RING finger gene that encodes a protein of 190 amino acids (Moilanen et al., 1998) and was originally isolated as an androgen receptorinteracting protein (Moilanen et al., 1998). Pero et al. (2001) found that the lack of RNF4 expression may play a role in the progression of testicular tumors implying that RNF4 is a growth inhibitor. RNF4 may involve differentiation and development by the finding that RNF4 interacts with paired type homeodomain protein goosecoid-like (gscl), a transcription factor involved in minimal DiGeorge critical region (Galili et al., 1998, 2000). Immunohistochemical analysis of mouse embryos revealed that RNF4 was expressed primarily in the developing nervous system and gonads (Yan et al., 2002). The sequence of RNF4 is highly conserved, exhibiting $91 \%$ identity between rat and human. RNF4 acts as a coactivator for steroid receptor-dependent transcription (Poukka et al., 2000), Sp1- (Saville et al., 2002), and TRPS1- (trichorhino-phalangeal syndrome) (Kaiser et al., 2003) mediated transcription.

In this report, the human GCH promoter was used to investigate the role of RNF4 as a protein cofactor modulating NF-Y function. After the dissection of GCH gene proximal promoter, we identified a novel mechanism for RNF4 on the activation of GCH gene transcription, that is, interacting with NF-Y on CCAAT box. These data indicate that RNF4 may enhance gene expression although functionally associated with NF-Y.

\section{Experimental Procedures}

Cell Culture. HEK293, HeLa, and SH-SY5Y cells were obtained from American Type Culture Collection (Manassas, VA) and maintained in Dulbecco's modified Eagle's medium supplemented with $10 \%$ fetal bovine serum (Invitrogen, Carlsbad, CA) in an incubator with $5 \% \mathrm{CO}_{2}$ at $37^{\circ} \mathrm{C}$. Drosophila melanogaster Schneider cells SL2 were kindly provided by Dr. Zhuang Z. L. (National Health Research Institute, Taiwan) and were grown in S2 insect medium (Invitrogen) supplemented with $10 \%$ fetal bovine serum at $25^{\circ} \mathrm{C}$.

Transfection, Reverse-Transcription, and Polymerase Chain Reaction (RT-PCR). For overexpression of RNF4 in SHSY5Y cells, LipofectAMINE 2000 (Invitrogen) were used for transfection according to the manufacturer's instruction. Plasmid pEGFP-N1 (BD Biosciences Clontech, Palo Alto, CA) was used as a control for transfection efficiency. By counting the number of GFP positive cells, a transfection efficiency of 60 to $70 \%$ could be obtained. SH-SY5Y cells were lysed with hypotonic buffer $(20 \mathrm{mM}$ Tris-HCl, $\mathrm{pH} 8.0,1 \mathrm{mM} \mathrm{MgCl}, 0.5 \%$ Nonidet P-40) for $5 \mathrm{~min}$ on ice $40 \mathrm{~h}$ after transfection. Cytosolic RNA was extracted using a mini-RNA extraction kit (Viogene, Taiwan). Endogenous GCH mRNA level was amplified using primers hGCH-F5 (5'-GCC TAC ACG TCC ATC CTG AG) and hGCH-R5 (5'-CAC CCA ACA TTG TGC TGG TC). The amplification cycle was $94^{\circ} \mathrm{C} 30 \mathrm{~s}, 60^{\circ} \mathrm{C} 30 \mathrm{~s}$, and $72^{\circ} \mathrm{C} 30 \mathrm{~s}$ for 30 cycles. Human $\beta$-actin was used as an internal for RT-PCR with primers h- $\beta$-actin-F, $5^{\prime}$-GGG TCA GAA GGA TTC CTA TG, and h- $\beta$-actin-R, $5^{\prime}$-GGT CTC AAA CAT GAT CTG GG and amplification cycle $94^{\circ} \mathrm{C} 30 \mathrm{~s}, 60^{\circ} \mathrm{C} 30 \mathrm{~s}$, and $72^{\circ} \mathrm{C} 30 \mathrm{~s}$ for 24 cycles. PCR cycle numbers were determined empirically for linear amplification of the RT products.

Plasmids Construction and Site-Directed Mutagenesis. The human GCH promoter 5.5-kb EcoRV/NcoI fragment was isolated from human genomic library (BD Biosciences Clontech) and was cloned into pCAT ${ }^{\mathrm{R}}$ 3-basic (called pRV-CAT hereafter). GCH promoter deletion clones pPst4.4-CAT, pPst2.8-CAT, pHindIII(-613)-
CAT, pSmaI(-285)-CAT, and $\mathrm{pEsp}(-205)-\mathrm{CAT}$ (translational start site was referred to as +1 ) were obtained by restriction digestion of pRV-CAT. pStuI(-1853)-CAT was obtained by removing the XhoI and StuI fragment from pPst2.8-CAT. CCAAT box-mutated construct mtCCAAT-CAT was generated by annealing oligonucleotides forward (5'-GATCCGGCCGATGGGAGCGCTCCGCGGCGCAGGCTGG) and reverse (5'- GATCCCAGCCTGCGCCGCGGAGCGCTCCCATCGGCCG) and was ligated into the BglII site of pEspI-CAT. AGP/EBP promoter A/E250-CAT containing upstream 250-bp promoter region was described elsewhere (Chang et al., 1995). AGP promoter AGP-CAT containing upstream 180-bp sequence was described elsewhere (Lee et al., 1993). GAL4-CAT and ARE-CAT were generous gifts from Dr. S.-C. Lee (Institute of BioMed, National Taiwan University, Taiwan) and Dr. M.-J. Tsai (Baylor College of Medicine, Houston, TX), respectively. To generate SmaI-Luc, CCAAT-Luc and mtCCAAT-Luc, the KpnI/NcoI fragments of pSmaICAT, pCCAAT-CAT, and mtCCAAT-CAT were inserted into the same sites in pGL3-basic vector (Promega, Madison, WI).

Full-length RNF4 cDNA (amino acids 1 to 190) was obtained by amplifying HeLa cDNA using primers RNF-F202 (5'-AGAGGGCATGAAAGGTTGAG), RNF4-F270 (5' -TAGGAAAGGAAAGGCACCAA), RNF4-R889 (5'-GTCTG TCCATCCGTCTCTCC), and RNF4-R930 (5'-CAGGAAAATGGAGGCAGATA) and PfuTurbo DNA polymerase (Invitrogen) and was cloned into pcDNA3.1 (Invitrogen). The Cterminal basic region-deleted RNF4 (RNF-NK, contains amino acids 1 to 177 ) was produced by amplification with primers BA270 (5'CGGGATCCTAGGAAAGGAAAGGCACCAA) and R890 (5'-GGAATTCT CACCTACAAGTTGGGCAAGT) using pcDNA-RNF4 as a template and was subcloned into pcDNA3.1-C. D. melanogaster cell expression vector $\mathrm{pPac}-\mathrm{RNF} 4$ and $\mathrm{pPac}-\mathrm{RNF} 4-\mathrm{NK}$ was generated by ligating the BamHI/XhoI fragment of pcDNA-RNF4 and pcDNARNF4-NK into BamHI/XhoI of pPacO. pGEX-RNF4 was constructed by ligating the BamHI/PmeI fragment of pcDNA-RNF4 into the BamHI/SmaI site of pGEX-2T (Pfizer Inc., New York, NY). RNF4 C-terminal lysine and arginine mutants RNF4-KK178AA and RNF4KR183AA were generated by PCR using oligonucleotides mutated in Lys-178 and Lys-179 (KK178A-F, 5'-ACTTG TAGGGCAGCGATCAACCACAAA; KK178A-R, 5'-GTGGTTGATCGCTGCCCT ACAAGTTGG) and Lys-183 and Arg-184 (KR183A-F, 5'-ATCAACCACGCAGCG TACCACCCCATT; KR183A-R, 5'-GGGGTGGTACGCTGCGTGGTTGATCTT). The products were digested with BamHI/ EcoRI and ligated into pcDNA 3.1-C. All constructs were verified by sequencing. pFLAG-RNF4 and pFLAG-RNF4-NK containing FLAGepitope were constructed by ligating the EcoRI/XhoI fragment from pcDNA3.1-RNF4 and the BamHI/EcoRI fragment from pcDNA3.1RNF4-NK into the pCMV-Taq2B and pCMV-Taq2A, respectively (Stratagene, La Jolla, CA). The BamHI/XhoI fragment from pcDNA3.1-RNF4 and the BamHI/EcoRI fragment from pcDNA3.1RNF4-NK were inserted into the same sites in pREST (Invitrogen). CMV driven GAL4-DNA binding domain fusion protein expression vector, pFA-RNF4, was constructed by ligating the BamHI/PmeI fragment of pcDNA3.1-RNF4 into the BamHI/SmaI site of pFA-CMV (Stratagene). GAL4-reporter pFR-Luc was obtained from Stratagene. pHA was constructed with PCR method using primer $5^{\prime}$ GTTAAGCTTACCATGGCTTACCCATACGATGTTCCT GACTATGCGGGCTATCCCTATGACGTCCCGGACTATGCAGGATCCCC and cloned into HindIII/BamHI site of pcDNA3.1/myc/His, which contains two HA epitopes. pHA-RNF4, pHA-RNF4-NK were constructed by ligating BamHI/EcoRI fragment of pcDNA3.1-RNF4 or pcDNA3.1RNF4-NK into pHA. Sequences and reading frame were verified by sequencing. Eukaryotic expression vector NF-YA, NFYB, NF-YC, and the dominant-negative vector NF-YAm19 were generously provided by Dr. R. Mantovani (University of Modena Reggio, Modena, Italy). D. melanogaster cell expression plasmids pPacNF-YA, pPacNF-YB, and pPacNF-YC were generously provided by Dr. T. F. Osborne (Department of Molecular Biology and Biochemistry, University of California, Irvine). 
Transfection and Western Blot Analysis. Calcium phosphate precipitation method was used for transfection (Graham and van der $\mathrm{Eb}, 1973)$. HEK293 cells in 6-cm dishes were transfected with a combination of $0.5 \mu \mathrm{g}$ of pGCH-CAT, 0.1 to $0.2 \mu \mathrm{g}$ of pcDNA-RNF4, $0.05 \mu \mathrm{g}$ of pCH110 (Promega) and pcDNA3.1 to give same total amount of DNA in each assay. Cells were subjected to mRNA extraction, CAT activity determination, or Western blot analysis $40 \mathrm{~h}$ after transfection. Western blot analysis was performed with a GCH antiserum (Hwu et al., 1999) by standard methods (Harlow, 1988). Blots were developed using the enhanced chemiluminescence detection system (Amersham Biosciences Inc., Piscataway, NJ). $\beta$-Galactosidase activity was included as an internal control to normalize transfection efficiency.

Expression of Recombinant Proteins and Production of RNF4 Antibody. Recombinant RNF4 (amino acid 89-190) protein was expressed by His-tagged vector pRSET in Escherichia coli BL21(DE3) cells and purified by Ni column according to the manufacturer's instruction (Novagen, Madison, WI). RNF4 antibody was raised by immunizing New Zealand White rabbits with the recombinant protein. pRSETA-RNF4 and pRSETB-RNF4-NK were expressed and purified from the soluble fraction of $E$. coli BL21(DE3) lysates.

Nuclear Extract Preparation and Electrophoretic Gel Mobility Shift Assay (EMSA). Preparation of nuclear extracts from HEK293 and SH-SY5Y cells was conducted as described by Lee et al. (1996). The labeled probe $\left(2.5 \times 10^{4} \mathrm{cpm}\right)$ was incubated with purified recombinant RNF4 and RNF4-NK and $2 \mu \mathrm{g}$ of poly(dI)-poly(dC) in reaction buffer $(3 \mathrm{mM}$ HEPES, $\mathrm{pH} 7.9,3.2 \mathrm{mM} \mathrm{KCl}, 0.02 \mathrm{mM}$ EDTA, $0.1 \mathrm{mM}$ dithiothreitol, $0.16 \mathrm{mM} \mathrm{MgCl}_{2}$ and $10 \%$ glycerol). The binding mixtures were incubated in room temperature for $30 \mathrm{~min}$ and loaded on $1 \%$ agarose gel. The CCAAT box probe was synthesized by amplifying pHindIII-CAT with primers GCH-CAT-F-235 (5'-GATCCGGCCAATGGGAGCGCTCCGCGGCGCAGGCTGA), and CAT-R201 (5'-CATCAGGCGGGCAAGAATGTG) (Lee and Lee, 2003) to generate a probe 436 -bp in length. The mutant CCAAT box probe was synthesized by amplifying pCCAAT-mt-CAT with primers GCHCCAATmut-F-235 (5'-GAT CCG GAT GGG AGC GCT CCG CGG CGC AGG CTG G) and CAT-R201. Double-stranded oligonucleotide GCH-Sp1-CCAAT (5'-GATCGAGGCGGGGCCGGCCAA TGGGAGCGCTCCGCGGCGCAGGC, 43-bp in length), Sp1 (5'-GATCATTCGATCGGGGCGGGGCGAGC, 26-bp in length), and CCAAT box (5'GATCCGGCCAATGGGAGCGCTCCGCGGCGCAGGCTGA, 37-bp in length) were also used in EMSA. NF- $\kappa$ B, GCF (GC box binding factor), and consensus CCAAT oligonucleotides were synthesized as described previously (Lee et al., 1996; Kapatos et al., 2000).

Expression and Purification of GST-RNF4 Fusion Protein. Proteins were expressed in E. coli BL21(DE3) cells. Three hours after induction of the $E$. coli with isopropyl $\beta$-D-thiogalactoside, the bacteria were homogenized in phosphate-buffered saline $(140 \mathrm{mM} \mathrm{NaCl}$, $2.7 \mathrm{mM} \mathrm{KCl}, 10 \mathrm{mM} \mathrm{Na}_{2} \mathrm{HPO}_{4}, 1.8 \mathrm{mM} \mathrm{KH}_{2} \mathrm{PO}_{4}, \mathrm{pH} 7.3$ ), and the expressed protein was purified from this homogenate by binding the fusion protein to glutathione-Sepharose 4B beads according to the manufacturer's instruction (Amersham Biosciences Inc.). The purity of the isolated fusion protein was assessed by gel electrophoresis. The binding buffer contained $50 \mathrm{mM}$ Tris-HCl, pH 7.8, $150 \mathrm{mM} \mathrm{KCl}$, $10 \%$ glycerol, $0.5 \mathrm{mM}$ EDTA, $0.1 \%$ Nonidet P-40, $5 \mathrm{mM} \mathrm{MgCl}$, 0.5 $\mathrm{mM}$ phenylmethylsulfonyl fluoride, $2 \mathrm{mg} / \mathrm{ml}$ aprotinin, and $3 \mathrm{mg} / \mathrm{ml}$ pepstatin A.

Coimmunoprecipitation Assay. pFLAG-RNF4 and NF-YB were transfected into HEK293 cells. Forty hours after transfection, the cells was lysed in binding buffer, centrifuged, and subjected to immunoprecipitation with M2 beads (Sigma-Aldrich, St. Louis, MO) that recognize FLAG epitope. The beads were washed four times with radioimmune precipitation assay buffer, boiled in sample loading buffer, and then separated by $12.5 \%$ SDS-polyacrylamide gel electrophoresis. Western blot analysis was performed with NF-Y antibody and detected with an enhanced chemiluminescence kit (Amersham Biosciences Inc.).
Chromatin Immunoprecipitation (ChIP) Assay. The ChIP assay was performed according to the manufacturer's recommendation (Upstate Biotechnology, Lake Placid, NY). In brief, histone was cross-linked to DNA by the addition of formaldehyde to a final concentration of $1 \%$, and cells were sonicated in $200 \mu \mathrm{l}$ of lysis buffer. One fourth of the total lysate was used for monitoring total DNA input, which was diluted 20 -fold in PCR. The rest of the lysate was cleared with $80 \mu \mathrm{l}$ of salmon sperm DNA/protein G-agarose slurry. Half the cleared lysate was incubated with HA beads (Roche Diagnostics, Indianapolis, IN) overnight at $4^{\circ} \mathrm{C}$. HA beads are agarose beads containing antibodies that recognize HA epitope. After reversing the cross-link, the eluted immunocomplex was digested with proteinase $\mathrm{K}$, and DNA was purified by phenol extraction. DNA was precipitated for detection by PCR with primers specific to the $\mathrm{GCH}$ promoter regions (i.e., GCH-CAT-F-235 and reversed primer, CATR201). PCR cycles were determined empirically. Amplified fragments were analyzed on a $1.2 \%$ agarose gel.

\section{Results}

Activation of GCH Promoter by RNF4. RNF4 was first identified as a cofactor for both androgen receptor-interacting protein (Moilanen et al., 1998) and Sp1 (Saville et al., 2002). In an attempt to explore the possible role of RNF4 in GCH gene expression, we isolated a 5.5-kb 5 '-flanking region of the human GCH gene. RNF4 strongly activates GCH reporter RV-CAT in transient transfection experiments (Fig. 1A). To test the specificity of RNF4 activation, a number of other promoters were also used, glucocorticoid-responsive element-containing promoter AGP-CAT, androgen-respon-

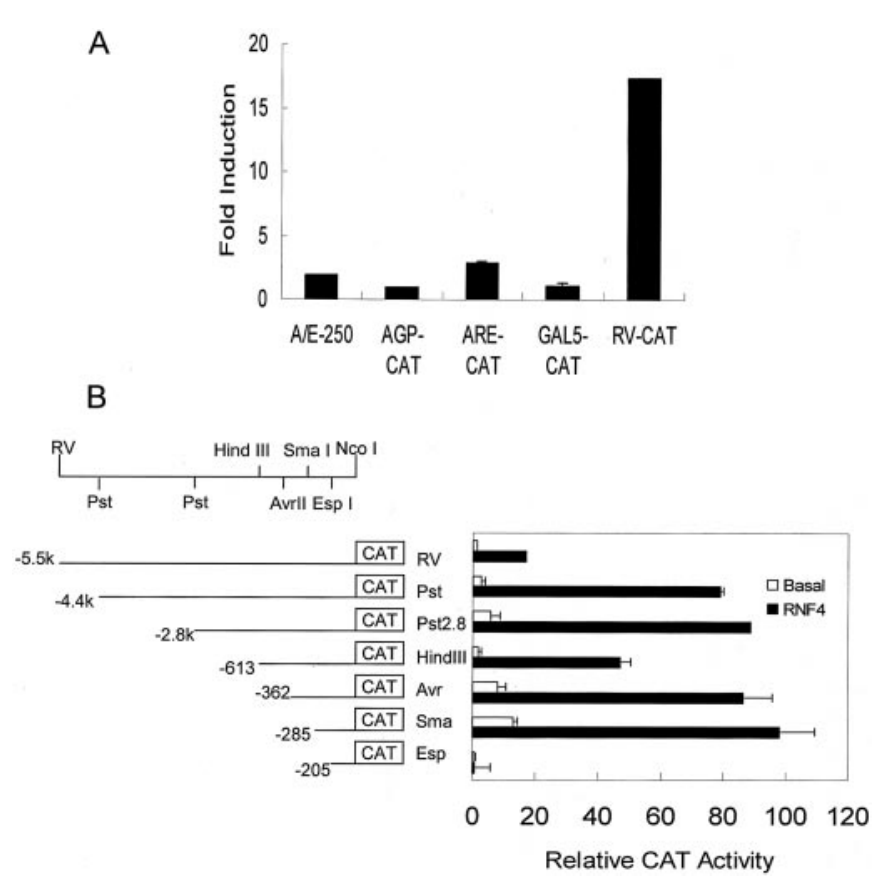

Fig. 1. RNF4 activates GCH gene expression. A, the 5.5-kb 5'-flanking region of human GCH gene (RV-CAT) was compared with other promoters, including A/E250-CAT, AGP-CAT, ARE-CAT, and GAL4-CAT for RNF4 activation. Each reporter was cotransfected with $0.2 \mu \mathrm{g}$ of pcDNA3.1C or pcDNA-RNF4 in HEK293 cells. Fold induction was calculated for each promoter individually. B, mapping of the cis-element for RNF4 activation on GCH promoter. A schematic view of the promoter constructs is on the left. These plasmids were transfected together with $0.2 \mu \mathrm{g}$ of pcDNA3.1C (open bars) or pcDNA-RNF4 (gray bars) into HEK293 cells, and CAT activities were measured and represented as percentage of CAT conversion. We repeated the experiments three times with similar results, and the values were from three independent experiments. Error bars indicated one standard deviation. 
sive element reporter ARE-CAT, and GAL4-CAT, but little activation was observed. Among these reporters, 2- to 3-fold induction was observed in Sp1-containing promoter A/E250CAT (Chang et al., 1995), which is similar to the findings by Moilanen et al. (1998).

cis-Elements Responsible for RNF4 Induction. To determine the cis-elements on the 5 '-flanking region of human $\mathrm{GCH}$ promoter that contribute to the induction by RNF4, a serial deletion of the $5.5-\mathrm{kb} \mathrm{GCH}$ promoter was done. These 5 '-deletion constructs (Fig. 1B, left), including $\mathrm{pRV}(-5.5 \mathrm{k})$ CAT, pPst (-4.4k)-CAT, pPst(-2.8k)-CAT, pHindIII(-613)-CAT, pAvrII(-362)-CAT, pSmaI(-285)-CAT, and pEspI(-205)-CAT, were transfected together with pcDNA-RNF4 into HEK293 cells. As shown in Fig. 2, all constructs except pEspI(-205)CAT showed considerable induction by RNF4 (Fig. 1B, right). Therefore, the 81-bp region between $\mathrm{SmaI}$ and EspI (nucleotide -285 to -205 ) on the GCH promoter is important for the activation by RNF4.

RNF4 Activates GCH Promoter through a CCAAT Box. Searching transcription factor binding sites using Signal Scan (http://bimas.dcrt.nhri.gov) on DNA sequence between nt-285 and nt-205 revealed three consensus Sp1 binding sites, one CRE and one CCAAT box (Fig. 2A). These binding sites have been described by Kapatos et al. (2000). To further clarify the role of these elements for RNF4-mediated activation, constructs containing either one or two of the three transcription factor binding sites were tested. As shown in Fig. 2A, 3xSp1-CAT (containing three Sp1 sites) lost inducibility. In contrast, Sp1-CCAAT-CAT (containing one Sp1 and one CCAAT box) and CCAAT-CAT (containing one CCAAT box) both maintained high inducibility. Hirayama has shown that transcription factors including NF-Y and ATF2 but not Sp1-4 bind to this region by DNase I footprinting analysis and EMSA (Kapatos et al., 2000;

A

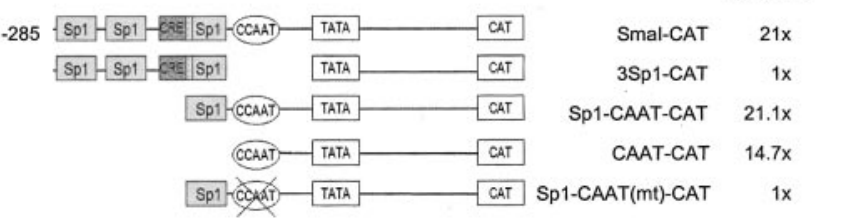

B

\begin{tabular}{|c|c|c|}
\hline-287 & $\frac{\text { CdGGGGG CGCGAGGCCG AGGGGCGCGG }}{\mathrm{Sp} 1}$ & dGAGGGCCGT \\
\hline-250 & $\frac{\text { GACGCGAGGC GGGGCCGGCC AATGGGAGCG }}{\text { CRE }} \frac{\text { Sp1 }}{\text { CCAAT }}$ & CTCCGCGGCG \\
\hline-210 & CAGGCTGAGC CGCCGGGCCA TAAAAAGGAG & GCGCGGCCGG \\
\hline-170 & GCTTTCCAGC TTGTGGCCGC TCCCGGCTCG & GAGTGTGATC \\
\hline-130 & TAAGCAGGTC GCGTACCTTC CTCAGGTGAC & TCCGGCCACA \\
\hline-90 & GCCCATTGTC CGCGGCCACC GGCGGAGTTT & AGCCGCAGAC \\
\hline-50 & CTCGAAGCGC CCCGGGGTCC TTCCCGAACG & GCAGCGGCTG \\
\hline-10 & $\begin{array}{r}\text { CGGCGGGTCC ATG } \\
+1\end{array}$ & \\
\hline
\end{tabular}

Fig. 2. A proximal CCAAT box is essential for RNF4-enhanced GCH gene transcription. A, fold activation of different GCH promoter constructs by RNF4 (pcDNA-RNF4) in HEK293 cells. The structures of the constructs are depicted on the left. SmaI-CAT was used as a positive control. We repeated the experiments four times, and the values were from three independent experiments. B, nucleotide sequence of the 5 '-flanking region of human GCH gene. Putative protein binding sites are boxed or underlined. Numbering is according to the distance from the translation initiation site (ATG as +1).
Hirayama et al., 2001). We mutated consensus NF-Y binding site CCAAT to CCGAT, which abolished NF-Y binding but not CCAAT enhancer-binding protein binding (Lee et al., 1993), and the activation by RNF4 was lost (Fig. 2A). These data suggest that RNF4-enhanced GCH proximal promoter activity is dependent on this CCAAT box. A GCH 5'-flanking sequence indexed relative to the translation start site was shown in Fig. 2B.

RNF4 Binds to the CCAAT Box. We performed EMSA using HEK293 nuclear extracts along with the doublestranded oligonucleotide CCAAT-Sp1 as a probe. A major shifted band was observed in the assay (Fig. 3A). The binding of this protein to CCAAT-Sp1 is specific, because unlabeled oligonucleotide corresponding to Sp1, NF- $\kappa \mathrm{B}$, or GCF binding sequences could not compete for the binding. However, when recombinant $\mathrm{RNF} 4$ protein was used in the assay, no binding to CCAAT-Sp1 could be detected at all (data not shown). The result is consistent with a previous report that RNF4 binds nonspecifically to DNA with longer stretches (Hakli et al., 2001). With a DNA fragment spanning from nt-235 to downstream +201 of pHindIII-CAT as a probe (translational start point as +1 ), recombinant RNF4 bound quite well (Fig. 3B). Although the intact CCAAT sequence is required for $\mathrm{GCH}$ activation, we found that RNF4 also bound to mutant CCAAT-containing DNA fragment (Fig. 3C). These data revealed that RNF4 binding to the CCAAT box is not sequencespecific.

Although the zinc finger domain and N-terminal NLS signal have been reported to be essential for RNF4 DNA binding (Poukka et al., 2000), we asked if the C terminus of RNF4, which is rich in basic residues (three Lys and one Arg within 13 amino acids) plays a role in DNA binding. We generated a C-terminal deletion clone RNF4-NK. The results showed that RNF4-NK bound to the 436-bp probe as the wild-type protein (Fig. 3B, lanes 5 and 6), which indicates that the $\mathrm{C}$ terminus is not necessary for DNA binding.

The C-Terminal Basic Residue-Rich Region of RNF4 Is Essential for Transactivation. Although RNF4 protein without the C-terminal basic region can bind to $\mathrm{GCH}$ promoter, we wanted to determine whether this region was important for RNF4-mediated activation. In the cotransfection experiment, RNF4-NK lost its ability to activate $\mathrm{GCH}$ promoter in HEK293 cells (Fig. 4B), although the protein expressions of RNF4 and RNF4-NK in cells were equivalent (Fig. 4C) and both proteins resided within the cell nuclei (data not shown).

We further mutated the basic residues within the $\mathrm{C}$ terminus. As shown in Fig. 4D, the transactivation abilities of both RNF4-KK178AA and RNF4-KR183AA were decreased, whereas that of RNF4-4KA (three lysines and one aspartic acid were all mutated to alanine) was lost completely (Fig. 4D). Multiple positively charged amino acids located in the $\mathrm{C}$ terminus of RNF4 protein play an essential role for its function in the activation of $\mathrm{GCH}$ promoter.

RNF4 Is a Coactivator for NF-Y. To verify whether RNF4 itself activates the basal transcriptional apparatus, RNF4 was ligated to the DNA-binding domain of GAL4 as pFA-RNF4. When pFA-RNF4 was transfected together with a GAL4 reporter, pFA-RNF4 could not activate GAL4 reporter. These data imply that RNF4 itself is not a transactivator (Fig. 5A). NF-Y is known as a transcription activator for the CCAAT box. Because RNF4 itself does not show 
A

\section{Competitor}

- $\frac{\text { CAAT }}{50200} \frac{\mathrm{Sp} 1}{50200} \frac{\mathrm{NF}-\mathrm{kB}}{50200} \frac{\mathrm{GCF}}{50200}$

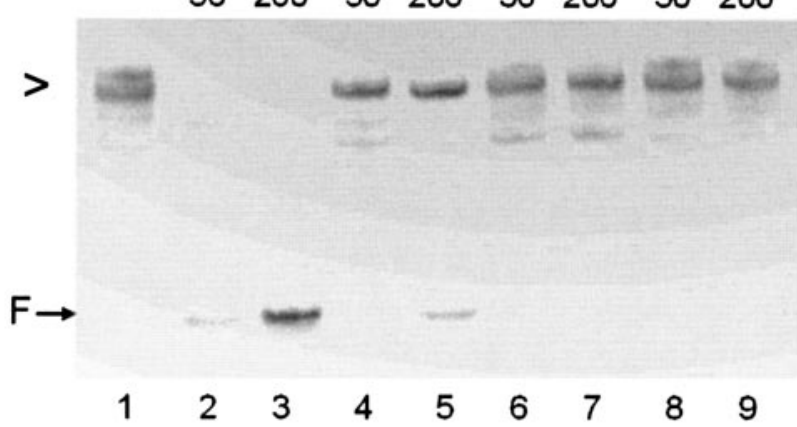

B

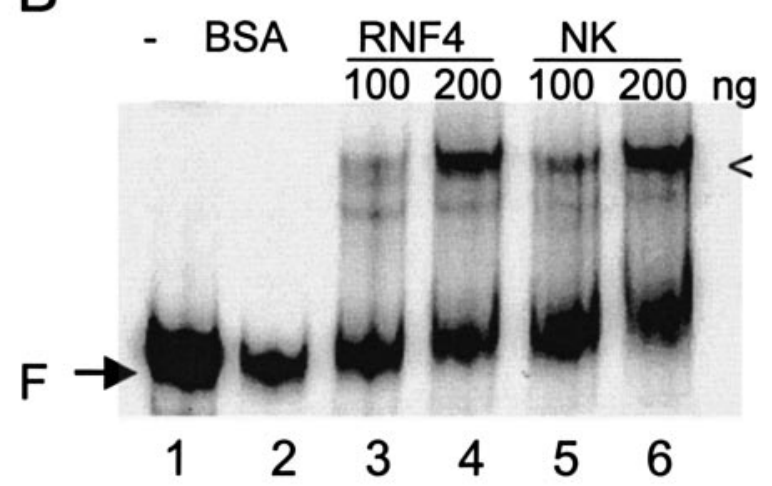

C
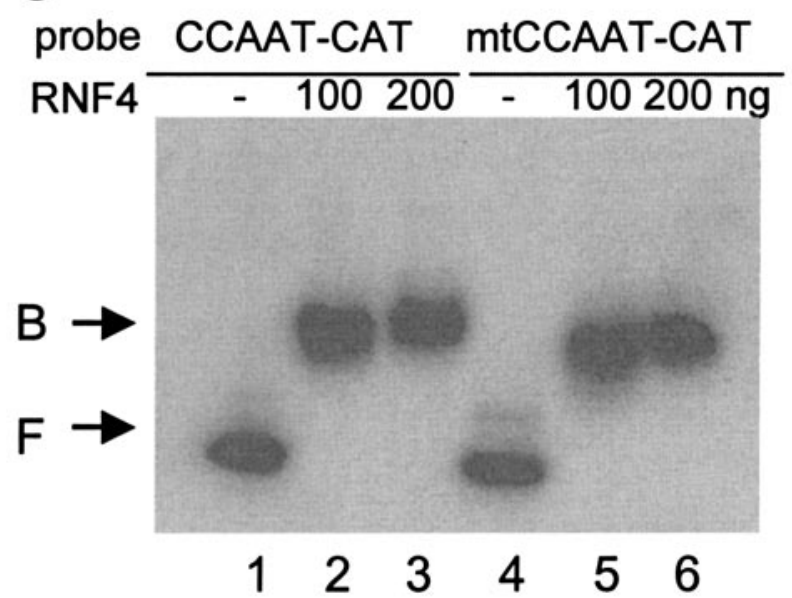

Fig. 3. Analysis of RNF4 binding to the GCH promoter. A, EMSA using synthetic CCAAT box oligonucleotide as a probe. End-labeled probe was incubated with $2 \mu \mathrm{g}$ of HEK293 nuclear extract in each lane. A major shifted band was observed (arrowhead, lane 1). Molar excess (50- or 200 -fold) of unlabeled competitor CCAAT box, Sp1, NF- $\kappa \mathrm{B}$, or GCF were also added (lanes 2-9). F, free probe. B, EMSA with the 436-bp (-235 to +201 of pHindIII-CAT) GCH probe was performed. Either bovine serum albumin (BSA, lane 2), recombinant RNF4 (lanes 3 and 4), or RNF4-NK (lanes 5 and 6) was incubated with the probe. C, EMSA with the 436-bp GCH probe with normal (lanes 1-3) or mutant CCAAT (lanes 4-6) sequences. The probe was incubated with none (lanes 1 and 4), $100 \mathrm{ng}$ (lanes 2 and 5), or $200 \mathrm{ng}$ (lanes 3 and 6) of the recombinant RNF4 protein. B, DNA-protein complex. We repeated the experiments three times with similar results, and one was used as representative.
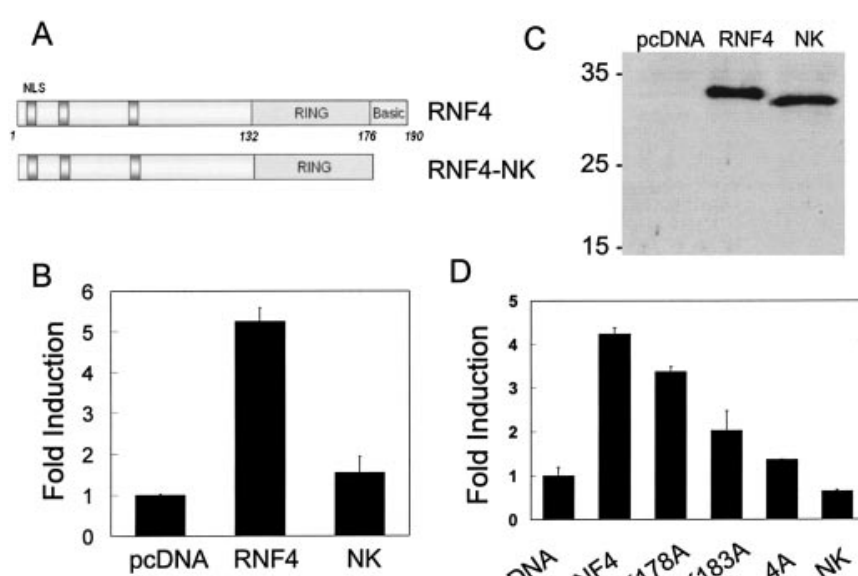

D

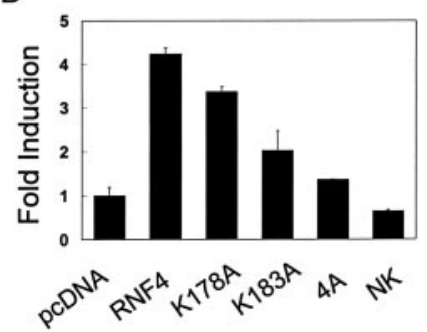

Fig. 4. C-terminal basic region of RNF4 is essential to activate GCH transcription. A, schematic diagram of the deletion clones. B, relative luciferase activity of HEK293 cells cotransfected with either $0.2 \mu \mathrm{g}$ of SmaI-Luc, $20 \mathrm{ng}$ of pcDNA-RNF4 (RNF4), pcDNA-NK (NK), or pcDNARNF4-NR (NR). C, Western blot with anti-RNF4 antibody. D, effect of RNF4 mutants on transcription of CCAAT reporter. CCAAT-Luc was transfected together with either RNF4, RNF4-KK178AA (K178A), RNF4KR183AA (K183A), RNF4-4KA (4A), RNF4-NK (NK), or RNF4-NR (NR) in HEK293 cells. Relative luciferase activity was expressed in comparison to pcDNA3.1. We repeated the experiments four times with similar results, and one was used as representative.
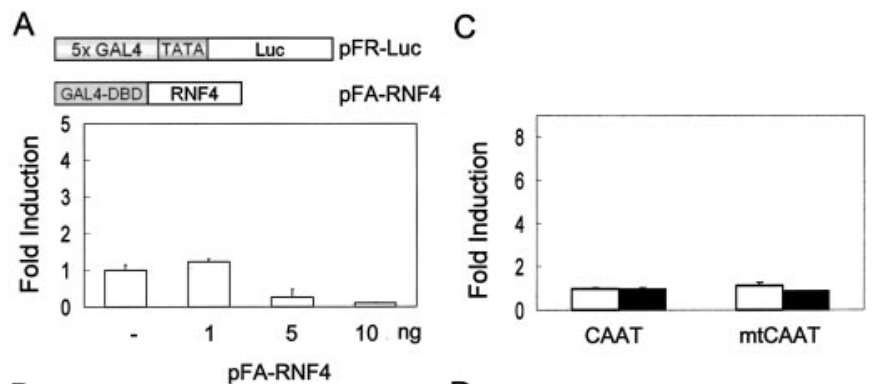

$\mathrm{B}$
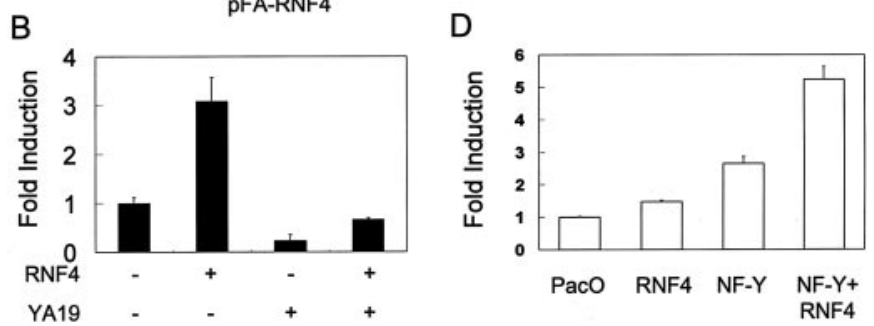

Fig. 5. RNF4 is a coactivator for NF-Y. A, schematic view of pFR-Luc and pFA-RNF4. Fold induction of the luciferase activity by pFA-RNF4 is shown. HEK293 cells were transfected with $200 \mathrm{ng}$ of pFR-Luc, and 1, 5 or $10 \mathrm{ng}$ of pFA-RNF4. B, dominant-negative NF-YAm19 (YA19) inhibits the basal transcription and RNF4-mediated activation of CCAAT-CAT reporter. HEK293 cells were transiently transfected with $0.5 \mu \mathrm{g}$ of CCAAT-CAT, $0.1 \mu \mathrm{g}$ of NF-YAm19 (YA19), and $0.2 \mu \mathrm{g}$ of pcDNA-RNF4. C, SL2 cells were cotransfected with $0.5 \mu \mathrm{g}$ of CCAAT-Luc or mtCCAATLuc with (gray boxes) or without (white boxes) $0.1 \mu \mathrm{g}$ of pPac-RNF4. D, SL2 cells were transfected with $0.5 \mu \mathrm{g}$ of CCAAT-Luc with $0.1 \mu \mathrm{g}$ of pPac-RNF4 or $0.1 \mu \mathrm{g}$ of pPacNF-Y (including pPacNF-YA, pPacNF-YB, and pPacNF-YC) plasmids. CAT or luciferase activities relative to the un-induced CCAAT construct were depicted. Error bars indicate one standard deviation. We repeated the experiment three times with similar results, and one was used as representative. 
binding specificity to the CCAAT box (Fig. 4), it is likely that RNF4 is a coactivator of NF-Y. RNF4 activated pCCAATCAT (Fig. 2A) in HEK293 cells that contain endogenous NF-Y. The NF-Y-dependent activation by RNF4 was then examined by introducing a dominant-negative NF-Y mutant, NF-YAm19 into the HEK293 cells. The results revealed that NF-YAm19 abolished RNF4-mediated activation of the CCAAT box in HEK293 cells (Fig. 5B). We further examined the RNF4 activity using $D$. melanogaster SL2 cells that are devoid of endogenous NF-Y. As shown in Fig. 5C, RNF4 could not activate the CCAAT reporter activity in SL2 cells. Moreover, when we cotransfected RNF4 and NF-Y in SL2 cells, NF-Y activated CCAAT reporter, and RNF4 and NF-Y activated CCAAT reporter synergistically (Fig. 5D). As a whole, these results suggest that RNF4 activates human GCH gene through a functional interaction with NF-Y or as a coactivator of NF-Y.

RNF4 Interacts with NF-Y. To prove whether RNF4 interacts with NF-Y, immunoprecipitation and GST pulldown experiments were done. In immunoprecipitation experiments, NF-Y can be coprecipitated with FLAG-tagged RNF4 (Fig. 6); however, using $\left[{ }^{35} \mathrm{~S}\right]$ methionine, in vitro transcription/translated NF-Y subunits, neither NF-YA nor NF-YB could be pulled down by GST-RNF4 (data not shown). NF-YC was not available for the assay.

RNF4 Activates Endogenous GCH Gene Expression. To investigate whether RNF4 activates endogenous GCH gene expression, transient transfection experiments were performed in a neuroblastoma cell line SH-SY5Y. The result showed that overexpression of RNF4 but not RNF4-NK elevated endogenous GCH mRNA levels (Fig. 7A). To examine whether RNF4 binds to endogenous GCH promoter, we performed chromatin immunoprecipitation assay. As shown in
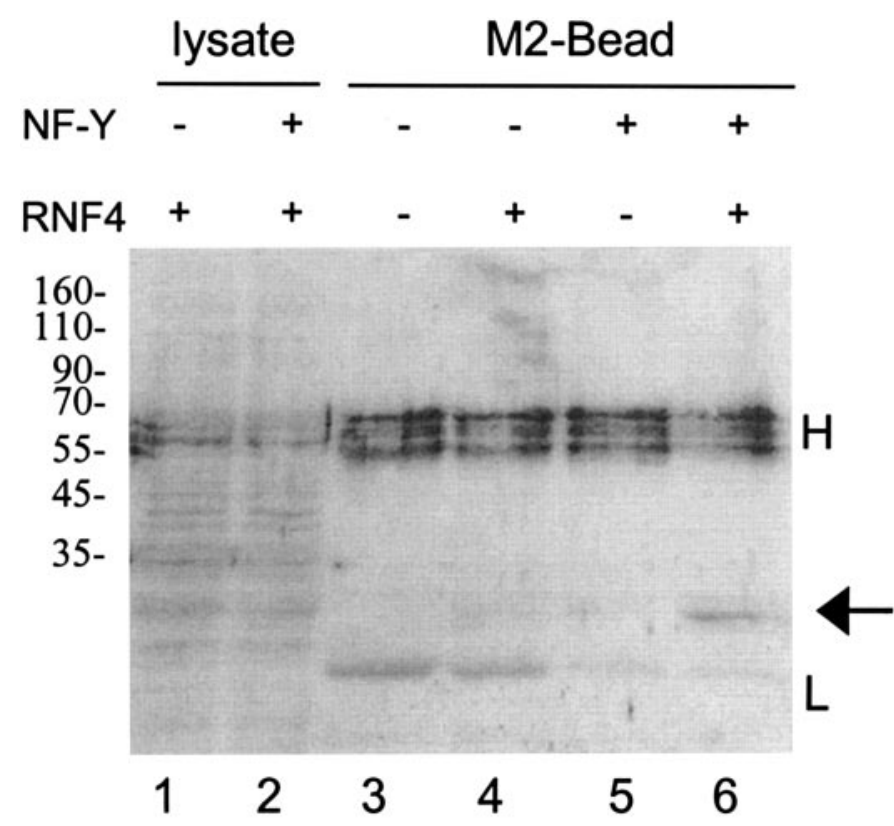

Fig. 6. RNF4 interacts with NF-Y. Immunoprecipitation experiment: HEK293 cells were transfected with $2 \mu \mathrm{g}$ each of pFLAG-RNF4 and NF-Y expression vectors; cell extracts were harvested and incubated with M2 beads. The immunoprecipitates were separated by SDS-polyacrylamide gel electrophoresis, and Western blot was probed by anti-NF-Y antibody. $\mathrm{H}$ and $\mathrm{L}$ indicate the heavy and light chain of immunoglobin from $\mathrm{M} 2$ beads. Arrow indicates NF-Y. We repeated the experiments four times with similar results, and one was used as representative.
Fig. 7B, RNF4 binds to the CCAAT box of the GCH gene (lane 4). We conclude that RNF4 and NF-Y association to the GCH promoter correlates to the transcriptional activation of the gene.

\section{Discussion}

Both RNF4 and GCH are enriched in the brain (Moilanen et al., 1998). This is the first report demonstrating RNF4 transactivation through the CCAAT box. We showed that RNF4 coactivated with NF-Y on GCH proximal promoter because RNF4 does not have sequence specificity for CCAAT box. Evidence for this conclusion came from several experiments. First, RNF4 lost the activation on CCAAT box in SL2 cells, which lack endogenous NF-Y. Second, dominant-negative NF-YA (NF-YAm19) abolished RNF4-mediated activation on CCAAT box. We also demonstrated the RNF4 physical interaction between RNF4 and NF-Y. Last, we introduced RNF4 into a neuroblastoma cell line and observed the elevation of endogenous GCH mRNA levels, and the interaction between RNF4 and endogenous GCH promoter in chromatin immunoprecipitation. Our findings not only provide a molecular basis for GCH regulation but also extend the knowledge about the mechanism of RNF4-mediated gene activation.

CCAAT box is one of the most ubiquitous DNA binding sequences, presented in more than $30 \%$ of eukaryotic promoters (Bucher, 1990) in housekeeping, inducible, cell cycleregulated, and developmentally controlled genes (Berry et
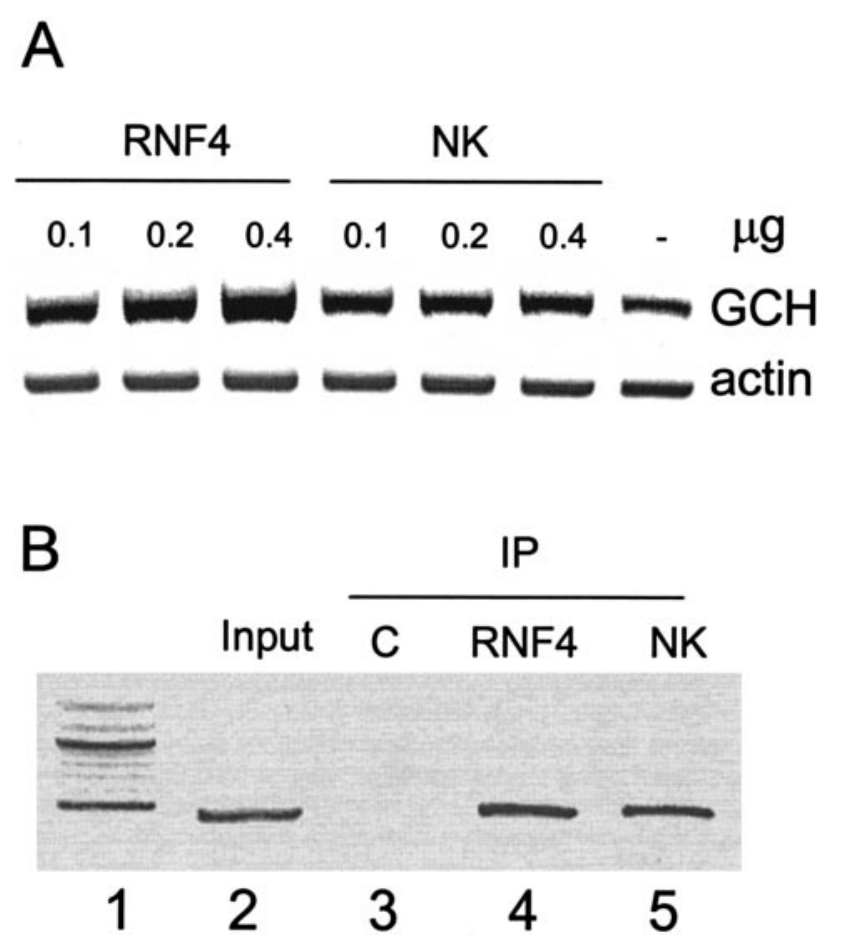

Fig. 7. RNF4 activates GCH gene expression in vivo. A, SH-SY5Y neuroblastoma cells were transiently transfected with $0.1,0.2$, or $0.4 \mu \mathrm{g}$ of either pcDNA-RNF4 or pcDNA-RNF4-NK with $0.1 \mu \mathrm{g}$ of pEGFP-N1. Cells were harvested, and RT-PCR was performed for GCH and actin $40 \mathrm{~h}$ after transfection. B, ChIP assays were performed as described under Materials and Methods. HEK293 cells (10-cm dish) were transfected with a GCH reporter (CCAAT-CAT) and pHA-RNF4 expression vector. Input indicates the PCR product of total chromatin. HA beads were used to precipitate the RNF4-DNA complex. Control indicates the ChIP derived from the pHA transfected cells. We repeated the experiments five times with similar results, and one was used as representative. 
al., 1992; Roy and Lee, 1995; Ronchi et al., 1996; Marziali et al., 1997). CCAAT box binding factor, NF-Y is a ubiquitous transcription factor complex consisting of three subunits (A, $\mathrm{B}$, and $\mathrm{C}$ ). NF-Y has been implicated in the basal expression of catecholamine-synthesizing gene such as tryptophan hydroxylase (Cote et al., 2002). NF-Y and the CCAAT box are now shown to be involved in the expression of $\mathrm{GCH}$, a gene responsible for cofactor of catecholamine synthesis. Using DNase I footprinting analysis, Hirayama has demonstrated two protein binding domains on GCH promoter: an upstream Sp1-like site and a combined CRE-Sp1-CCAAT box element. EMSA and supershift assays demonstrated that the combined CRE-Sp1-CCAAT box element recruits ATF-2 and NF-Y but not Sp1-4 (Kapatos et al., 2000; Hirayama et al., 2001). These data suggested that human CRE-Sp1-CCAAT element attracts a large protein complex that would be able to distort DNA in this region. Our data obtained from ChIP further demonstrate that coactivator RNF4 plays a role in $\mathrm{GCH}$ expression through this region.

NF-YB and NF-YC contain a histone-like motif (Masiero et al., 2002; Romier et al., 2003), and NF-Y has been reported to be associated with histone acetyltransferase GCN5 and P/CAF (Currie, 1998). Coactivator p300, interacting with $\mathrm{NF}-\mathrm{Y}$ in vivo and in vitro, was reported to enhance NF-Y binding to the third CCAAT box on cyclin B2 promoter (Salsi et al., 2003). It has been proposed that p300 serves as a platform for NF-Y binding to the CCAAT box. NF-Y is able to recruit purified holo-TFIID in vitro and can associate with multiple $\mathrm{TAF}_{\mathrm{II}} \mathrm{S}$ on major histocompatibility complex class II Ea core element $(-115 /+60)$, which also contains three CCAAT boxes (Frontini et al., 2002). It's not clear if the same mechanisms work in promoters contain only one CCAAT box. RNF4 interacts efficiently with both supercoiled circular and four-way junction DNA. RNF4 has been shown to bind TBP (Moilanen et al., 1998), and it also recognizes nucleosomes (Hakli et al., 2001). Because RNF4 interacts with NF-Y, we propose that RNF4 may help the function of NF-Y when only one CCAAT box is present.

DNA context would be important in RNF4-mediated activation because when CCAAT box was moved to the downstream of CAT reporter, the CAT gene could not be activated

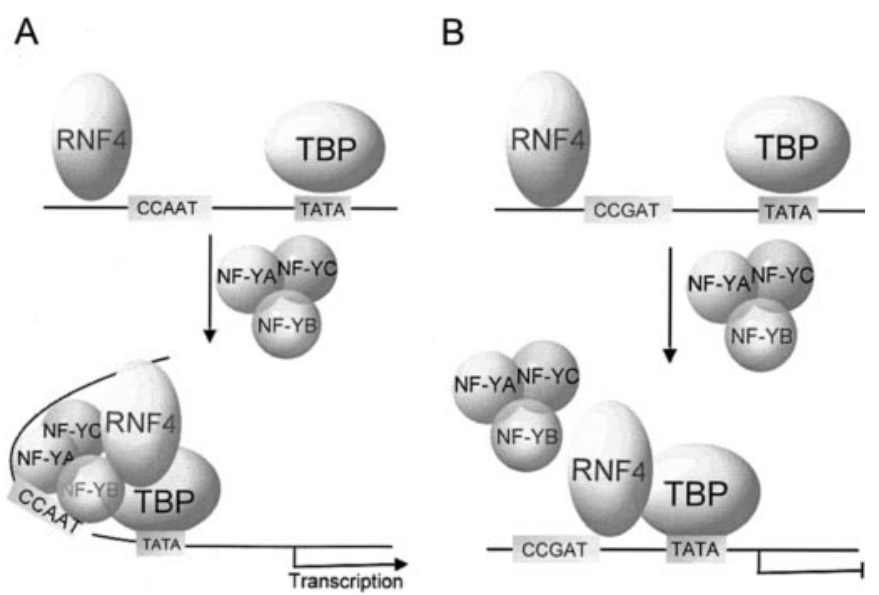

Fig. 8. A cartoon of RNF4-mediated activation. A, RNF4 binds to DNA and is brought to the CCAAT box through interaction with NF-Y, which binds to the box. The RNF4-NF-Y complex then acts on TBP. B, with a mutated CCAAT box, NF-Y cannot bind and RNF4 cannot be located to the CCAAT box. by RNF4 (data not shown). Because NF-YA is necessary for CCAAT box binding (Maity and de Crombrugghe, 1998) and RNF4-mediated GCH activation is dependent on NF-Y, we propose a model for RNF4-mediated activation of single CCAAT box: 1) RNF4 binds to DNA through its nonspecific DNA binding ability, 2) NF-Y binds to the CCAAT box, 3) RNF4 binds to NF-Y and thus is recruited to the CCAAT box, 4) the NF-Y-RNF4 complex alters chromatin configuration (Imbriano et al., 2001), and 5) RNF4-NF-Y complex binds to TFIID and recruits coactivators to activate gene expression. A schematic view of the model is shown in Fig. 8A. Figure 8B, in the presence of a mutated CCAAT box, NF-Y can not bind and there is no gene activation.

The nuclear localization signal of RNF4 located at its $\mathrm{N}$ terminus is essential for androgen receptor interaction (Moilanen et al., 1998). The C-terminal RING domain interacts with Sp1 through zinc finger (Hakli et al., 2001) and is essential for Sp1 activation (Poukka et al., 2000). RNF4 spanning amino acid region 29-129 lost TBP interaction ability (Moilanen et al., 1998). Our result showed that RNF4-NK ( $\Delta 177-190)$ retains DNA binding activity, and this is consistent with the previous finding (Hakli et al., 2001); however, RNF-NK cannot activate GCH promoter or the basic residues mutant anymore. Thus, the C-terminal basic region of RNF4 that is required for NF-Y-mediated gene activation may exist through the interaction with TBP.

We are not sure why RNF4 is enriched in non-DN cells. The balance between protein production, stability, and degradation should be critical. We have found that the expression of GCH is highly dependent on chaperones (Hwu et al., 2004). Female patients are more effected by HPD/DRD (Ichinose et al., 1994). Because RNF4 interacts with steroid receptors such as androgen receptor and estrogen receptor (Poukka et al., 2000; Saville et al., 2002), it will be very interesting to know if RNF4 is responsible for sex difference in GCH gene expression and female preference in HPD/DRD. Further investigations are needed.

\section{Acknowledgments}

We are grateful to Dr. M.-J. Tsai (Baylor College of Medicine, Houston, TX) for human androgen receptor and Dr. T. F. Obsorne (Department of Molecular Biology and Biochemistry, University of California, Irvine) for D. melanogaster NF-Y expression plasmids NF-YA, NF-YB, and NF-YC. We thank Chun-Jen Wang and Mei-Yi Leu for excellent technical help.

\section{References}

Berry M, Grosveld F, and Dillon N (1992) A single point mutation is the cause of the Greek form of hereditary persistence of fetal haemoglobin. Nature (Lond) 358: 499-502.

Blau N and Niederwieser A (1985) GTP-cyclohydrolases: a review. J Clin Chem Clin Biochem 23:169-176.

Bucher P (1990) Weight matrix descriptions of four eukaryotic RNA polymerase II promoter elements derived from 502 unrelated promoter sequences. $\mathrm{J} \mathrm{Mol} \mathrm{Biol}$ 212:563-578.

Chang CJ, Shen BJ, and Lee SC (1995) Autoregulated induction of the acute-phase response transcription factor gene, agp/ebp. DNA Cell Biol 14:529-537.

Cote F, Schussler N, Boularand S, Peirotes A, Thevenot E, Mallet J, and Vodjdani G (2002) Involvement of NF-Y and Sp1 in basal and cAMP-stimulated transcriptional activation of the tryptophan hydroxylase (TPH) gene in the pineal gland J Neurochem 81:673-685.

Currie RA (1998) NF-Y is associated with the histone acetyltransferases GCN5 and P/CAF. J Biol Chem 273:1430-1434.

Frontini M, Imbriano C, diSilvio A, Bell B, Bogni A, Romier C, Moras D, Tora L, Davidson I, and Mantovani R (2002) NF-Y recruitment of TFIID, multiple interactions with histone fold TAF(II)s. J Biol Chem 277:5841-5848.

Galili N, Epstein JA, Leconte I, Nayak S, and Buck CA (1998) Gscl, a gene within the minimal DiGeorge critical region, is expressed in primordial germ cells and the developing pons. Dev Dyn 212:86-93. 
Galili N, Nayak S, Epstein JA, and Buck CA (2000) Rnf4, a RING protein expressed in the developing nervous and reproductive systems, interacts with Gscl, a gene within the DiGeorge critical region. Dev Dyn 218:102-111.

Graham FL and van der Eb AJ (1973) A new technique for the assay of infectivity of human adenovirus 5 DNA. Virology 52:456-467.

Hakli M, Karvonen U, Janne OA, and Palvimo JJ (2001) The RING finger protein SNURF is a bifunctional protein possessing DNA binding activity. J Biol Chem 276:23653-23660.

Harlow E (1988) Antibodies: A Laboratory Manual. Cold Spring Harbor Laboratory Press, Cold Spring Harbor, New York.

Hirano M, Yanagihara T, and Ueno S (1998) Dominant-negative effect of GTP cyclohydrolase I mutations in dopa-responsive hereditary progressive dystonia. Ann Neurol 44:365-371.

Hirayama K and Kapatos G (1998) Nigrostriatal dopamine neurons express low levels of GTP cyclohydrolase I protein. J Neurochem 70:164-170.

Hirayama K, Shimoji M, Swick L, Meyer A, and Kapatos G (2001) Characterization of GTP cyclohydrolase I gene expression in the human neuroblastoma SKN$\mathrm{BE}(2) \mathrm{M} 17$ : enhanced transcription in response to cAMP is conferred by the proximal promoter. J Neurochem 79:576-587.

Hwu WL, Chiou YW, Lai SY, and Lee YM (2000) Dopa-responsive dystonia is induced by a dominant-negative mechanism. Ann Neurol 48:609-613.

Hwu WL, Wang PJ, Hsiao KJ, Wang TR, Chiou YW, and Lee YM (1999) Doparesponsive dystonia induced by a recessive GTP cyclohydrolase I mutation. Hum Genet 105:226-230.

Ichinose H, Ohye T, Takahashi E, Seki N, Hori T, Segawa M, Nomura Y, Endo K, Tanaka H, Tsuji S, et al. (1994) Hereditary progressive dystonia with marked diurnal fluctuation caused by mutations in the GTP cyclohydrolase I gene. Nat Genet 8:236-242.

Imbriano C, Bolognese F, Gurtner A, Piaggio G, and Mantovani R (2001) HSP-CBF is an NF-Y-dependent coactivator of the heat shock promoters CCAAT boxes. $J$ Biol Chem 276:26332-26339.

Kaiser FJ, Moroy T, Chang GT, Horsthemke B, and Ludecke HJ (2003) The RING finger protein RNF4, a co-regulator of transcription, interacts with the TRPS1 transcription factor. J Biol Chem 278:38780-38785.

Kapatos G, Stegenga SL, and Hirayama K (2000) Identification and characterization of basal and cyclic AMP response elements in the promoter of the rat GTP cyclohydrolase I gene. J Biol Chem 275:5947-5957.

Lee PW and Lee YM (2003) Transcriptional regulation of mu opioid receptor gene by cAMP pathway. Mol Pharmacol 64:1410-1418.

Lee YM, Miau LH, Chang CJ, and Lee SC (1996) Transcriptional induction of the alpha-1 acid glycoprotein (AGP) gene by synergistic interaction of two alternative activator forms of AGP/enhancer-binding protein (C/EBP beta) and NF-kappaB or Nopp140. Mol Cell Biol 16:4257-4263.

Lee YM, Tsai WH, Lai MY, Chen DS, and Lee SC (1993) Induction of liver alpha-1 acid glycoprotein gene expression involves both positive and negative transcription factors. Mol Cell Biol 13:432-442.

Maity SN and de Crombrugghe B (1998) Role of the CCAAT-binding protein CBF/ NF-Y in transcription. Trends Biochem Sci 23:174-178.

Marziali G, Perrotti E, Ilari R, Testa U, Coccia EM, and Battistini A (1997) Transcriptional regulation of the ferritin heavy-chain gene: the activity of the CCAAT binding factor NF-Y is modulated in heme-treated Friend leukemia cells and during monocyte-to-macrophage differentiation. Mol Cell Biol 17:1387-1395.
Masiero S, Imbriano C, Ravasio F, Favaro R, Pelucchi N, Gorla MS, Mantovani R, Colombo L, and Kater MM (2002) Ternary complex formation between MADS-box transcription factors and the histone fold protein NF-YB. J Biol Chem 277:2642926435

Moilanen AM, Poukka H, Karvonen U, Hakli M, Janne OA, and Palvimo JJ (1998) Identification of a novel RING finger protein as a coregulator in steroid receptormediated gene transcription. Mol Cell Biol 18:5128-5139.

Nichol CA, Smith GK, and Duch DS (1985) Biosynthesis and metabolism of tetrahydrobiopterin and molybdopterin. Annu Rev Biochem 54:729-764.

Pero R, Lembo F, Di Vizio D, Boccia A, Chieffi P, Fedele M, Pierantoni GM, Rossi P, Iuliano R, Santoro M, et al. (2001) RNF4 is a growth inhibitor expressed in germ cells but not in human testicular tumors. Am J Pathol 159:1225-1230.

Poukka H, Aarnisalo P, Santti H, Janne OA, and Palvimo JJ (2000) Coregulator small nuclear RING finger protein (SNURF) enhances Sp1- and steroid receptormediated transcription by different mechanisms. J Biol Chem 275:571-579.

Romier C, Cocchiarella F, Mantovani R, and Moras D (2003) The NF-YB/NF-YC structure gives insight into DNA binding and transcription regulation by CCAAT factor NF-Y. J Biol Chem 278:1336-1345.

Ronchi A, Berry M, Raguz S, Imam A, Yannoutsos N, Ottolenghi S, Grosveld F, and Dillon N (1996) Role of the duplicated CCAAT box region in gamma-globin gene regulation and hereditary persistence of fetal haemoglobin. EMBO (Eur Mol Biol Organ) $J$ 15:143-149.

Roy B and Lee AS (1995) Transduction of calcium stress through interaction of the human transcription factor CBF with the proximal CCAAT regulatory element of the grp78/BiP promoter. Mol Cell Biol 15:2263-2274.

Salsi V, Caretti G, Wasner M, Reinhard W, Haugwitz U, Engeland K, and Mantovan R (2003) Interactions between p300 and multiple NF-Y trimers govern cyclin B2 promoter function. J Biol Chem 278:6642-6650.

Saville B, Poukka H, Wormke M, Janne OA, Palvimo JJ, Stoner M, Samudio I, and Safe S (2002) Cooperative coactivation of estrogen receptor $\alpha$ in ZR-75 human breast cancer cells by SNURF and TATA-binding protein. $J$ Biol Chem 277:24852497.

Shimizu S, Ishii M, Kawakami Y, Kiuchi Y, Momose K, and Yamamoto T (1999) Presence of excess tetrahydrobiopterin during nitric oxide production from inducible nitric oxide synthase in LPS-treated rat aorta. Life Sci 65:2769-2779.

Suzuki T, Ohye T, Inagaki H, Nagatsu T, and Ichinose H (1999) Characterization of wild-type and mutants of recombinant human GTP cyclohydrolase I: relationship to etiology of dopa-responsive dystonia. J Neurochem 73:2510-2516.

Werner ER, Werner-Felmayer G, Fuchs D, Hausen A, Reibnegger G, Yim JJ, Pfleiderer W, and Wachter H (1990) Tetrahydrobiopterin biosynthetic activities in human macrophages, fibroblasts, THP-1, and T 24 cells. GTP-cyclohydrolase I is stimulated by interferon-gamma and 6-pyruvoyl tetrahydropterin synthase and sepiapterin reductase are constitutively present. J Biol Chem 265:3189-3192.

Yan W, Hirvonen-Santti SJ, Palvimo JJ, Toppari J, and Janne OA (2002) Expression of the nuclear RING finger protein SNURF/RNF4 during rat testis developmen suggests a role in spermatid maturation. Mech Dev 118:247-253.

Address correspondence to: Dr. Yu-May Lee, Institute of Biological Chemistry, Academia Sinica, Taipei, Taiwan, Republic of China. E-mail: yml6120@gate.sinica.edu.tw. 